\title{
The Design of Digital All-Pass Filters Using Second-Order Cone Programming (SOCP)
}

\author{
S. C. Chan, H. H. Chen, and Carson K. S. Pun
}

\begin{abstract}
This brief proposes a new method for designing digital all-pass filters with a minimax design criterion using secondorder cone programming (SOCP). Unlike other all-pass filter design methods, additional linear constraints can be readily incorporated. The overall design problem can be solved through a series of linear programming subproblems and the bisection search algorithm. The convergence of the algorithm is guaranteed. Nonlinear constraints such as the pole radius constraint of the filters can be formulated as additional SOCP constraints using Rouche's theorem. It was found that the pole radius constraint allows an additional tradeoff between the approximation error and the stability margin. The effectiveness of the proposed method is demonstrated by several design examples and comparison with conventional methods.
\end{abstract}

Index Terms-All-pass digital filter, bisection, Rouche's theorem, second-order cone programming (SOCP).

\section{INTRODUCTION}

D IGITAL all-pass filters are useful in many applications such as digital communications, phase equalization, implementation of digital and multirate filters, etc. [1], due to their low implementation complexity and good numerical property. These attractive properties have motivated considerable research into the design of digital all-pass filters [2][3][4][5][6][7][8], which includes the weighted least-square designs in [3], [5], [6] and the minimax designs in [2], [4], [7], and [8].

In this brief, we propose a new method for designing causal-stable digital all-pass filters with a minimax design criterion using second-order cone programming $(\mathrm{SOCP})^{1} \mathrm{SOCP}$ is a special case of semidefinite programming (SDP) and it can be written as follows:

$$
\begin{aligned}
\min & \boldsymbol{b}^{T} \boldsymbol{x}, \\
\text { subject to } & \boldsymbol{d}_{i}^{T} \boldsymbol{x}+q_{i} \geq\left\|\boldsymbol{A}_{i} \boldsymbol{x}+\boldsymbol{c}_{i}\right\|_{2}, \quad i=1, \ldots, N
\end{aligned}
$$

where $\boldsymbol{x} \in R^{m}$ is the variable, $\boldsymbol{b}_{i}, \boldsymbol{d}_{i} \in R^{m}, \boldsymbol{c}_{i} \in R^{n_{i}}, q_{i} \in$ $R$ are constant vectors, and $A_{i} \in R^{n_{i} \times m}$ are constant matrices. Like SDP, SOCP is a convex programming problem and the global optimal solution is guaranteed, if it exists. Interested readers are referred to [10], [11], [16], [17], [19], [20], [21] for

Manuscript received April 20, 2004; revised June 30, 2004 and July 22, 2004 This paper was presented in part at the International Conference on Acoustics, Speech, and Signal Processing, Hong Kong, April 6-10, 2003. This paper was recommended by Associate Editor B. C. Levy.

The authors are with the Department of Electrical and Electronic Engineering, The University of Hong Kong, Hong Kong.

Digital Object Identifier 10.1109/TCSII.2004.840285

${ }^{1}$ The design problem can also be solved with SDP [22], which might offer more flexibility in imposing additional nonlinear constraints but requiring a longer running time. the design of finite-impulse response (FIR) and infinite-impulse response (IIR) filters using SDP and SOCP. Using the property of all-pass filters, we show that the minimax design problem of an all-pass filter can be cast as a quasi-convex constrained optimization problem and it can be solved through a series of LP subproblems and the bisection search algorithm. In addition, linear and possible convex quadratic constraints such as pole radius constraints can be readily incorporated as SOCP or in general SDP constraints. The effectiveness and flexibility of the proposed method are demonstrated by several design examples. It should be noted that if the magnitude response alone is of interest, then it is possible to formulate the IIR filter design problem as a SDP problem [19]. However, the filters so obtained might suffer from undesirable phase distortion. The phase-responses of all-pass-based filters, however, are approximately linear in the passbands. The brief is organized as follows. In Section II, a brief introduction to digital all-pass filters is given. Section III is devoted to the proposed SOCP design method. In Section IV, a design example and a detail comparison with other design techniques are given. Finally, conclusions are drawn in Section V.

\section{Digital All-Pass FiLters}

The transfer function of an $N$ th-order digital all-pass filter is

$$
H_{N}(z)=z^{-N} \frac{\sum_{n=0}^{N}\left(a_{r n}-j a_{i n}\right) z^{n}}{\sum_{n=0}^{N}\left(a_{r n}+j a_{i n}\right) z^{-n}}
$$

where $a_{r n}$ and $a_{i n}$ are, respectively, the real and imaginary parts of the complex-valued filter coefficients $a_{n}$. Substituting $z=$ $e^{j \omega}$ into (2.1), one gets the frequency response of $H_{N}(z)$ as $H_{N}\left(e^{j \omega}\right)=e^{j \theta(\omega)}$, where

$$
\begin{aligned}
\theta(\omega) & =-N \omega+2 \phi_{A}(\omega) \\
\tan \left(\phi_{A}(\omega)\right) & =\frac{\sum_{n=0}^{N}\left[a_{r n} \cdot \sin (n \omega)-a_{i n} \cos (n \omega)\right]}{\sum_{n=0}^{N}\left[a_{r n} \cdot \cos (n \omega)+a_{i n} \sin (n \omega)\right]} .
\end{aligned}
$$

To approximate a certain desired phase response $\theta(\omega)=\theta_{d}(\omega)$, we need to express the phase error $\theta_{e}(\omega)=\theta(\omega)$ $\theta_{d}(\omega)$ in terms of the filter coefficients as $e^{j \theta_{e}(\omega)}=$ $\sum_{n=0}^{N} a_{n}^{*} e^{j \Phi_{n}(\omega)} / \sum_{n=0}^{N} a_{n} e^{-j \Phi_{n}(\omega)}$, where $\Phi_{n}(\omega)=$ $n \omega-\left(N \omega+\theta_{d}(\omega)\right) / 2$. Therefore, we have

$$
\tan \left(\frac{\theta_{e}(\omega)}{2}\right)=\frac{\boldsymbol{a}_{r}^{T} \cdot \boldsymbol{s}(\omega)-\boldsymbol{a}_{i}^{T} \cdot \boldsymbol{c}(\omega)}{\boldsymbol{a}_{r}^{T} \cdot \boldsymbol{c}(\omega)+\boldsymbol{a}_{i}^{T} \cdot \boldsymbol{s}(\omega)}
$$

where

$$
\begin{aligned}
\boldsymbol{a}_{\theta} & =\left[\begin{array}{llll}
a_{\theta 0} & a_{\theta 1} & \ldots & a_{\theta N}
\end{array}\right]^{T}, \\
\boldsymbol{c}(\omega) & =\left[\begin{array}{llll}
\cos \left(\Phi_{0}(\omega)\right) & \cos \left(\Phi_{1}(\omega)\right) & \ldots & \cos \left(\Phi_{N}(\omega)\right)
\end{array}\right]^{T} \\
\boldsymbol{s}(\omega) & =\left[\begin{array}{llll}
\sin \left(\Phi_{0}(\omega)\right) & \sin \left(\Phi_{1}(\omega)\right) & \ldots & \sin \left(\Phi_{N}(\omega)\right)
\end{array}\right]^{T} .
\end{aligned}
$$




\section{PRoposed Design Method}

\section{A. Minimax Error Criterion}

If the all-pass filter is used to approximate a desired phase response over a set of disjoint intervals $\omega \in \Omega \subset[-\pi, \pi]$ in a weighted minimax error criterion, then the phase response error is to be bounded by a positive quantity $\Delta$. Since the tangent function is monotonic in the interval $[0, \pi / 2]$, the value of $\tan \left(\theta_{e}(\omega) / 2\right)$ is bounded above and below by $[-\delta, \delta]$, where $\tan (\Delta / 2)=\delta$. The design problem at hand is then given by

$$
\min _{\boldsymbol{a}} \delta
$$

$$
\text { subject to } \quad-\delta<W(\omega) \frac{\boldsymbol{a}_{r}^{T} \cdot \boldsymbol{s}(\omega)-\boldsymbol{a}_{i}^{T} \cdot \boldsymbol{c}(\omega)}{\boldsymbol{a}_{r}^{T} \cdot \boldsymbol{c}(\omega)+\boldsymbol{a}_{i}^{T} \cdot \boldsymbol{s}(\omega)}<\delta
$$

for $\omega \in \Omega \subset[-\pi, \pi]$, where $\boldsymbol{a}=\left[\boldsymbol{a}_{r}^{T}, \boldsymbol{a}_{i}^{T}\right]^{T}$ and $W(\omega)$ is a positive weighting function. If the denominator is positive, (3.1) becomes

$$
\begin{array}{cl}
\min _{\boldsymbol{a}} & \delta \\
\text { subject to } & -\delta\left[\boldsymbol{a}_{r}^{T} \cdot \boldsymbol{c}(\omega)+\boldsymbol{a}_{i}^{T} \cdot \boldsymbol{s}(\omega)\right] \\
& <W(\omega)\left[\boldsymbol{a}_{r}^{T} \cdot \boldsymbol{s}(\omega)-\boldsymbol{a}_{i}^{T} \cdot \boldsymbol{c}(\omega)\right] \\
& <\delta\left[\boldsymbol{a}_{r}^{T} \cdot \boldsymbol{c}(\omega)+\boldsymbol{a}_{i}^{T} \cdot \boldsymbol{s}(\omega)\right]
\end{array}
$$

for $\omega \in \Omega \subset[-\pi, \pi]$. This is possible because the denominator of (3.1) cannot change sign over the interested frequency band, otherwise, the ripple $\delta$ would become $\infty$ at some frequency. So the denominator is either of positive or negative sign. It can be shown that both cases give the same solution [16]. Thus, we only need to consider the case with positive denominator in (3.1). The importance of this result is that we can transit from (3.1) to (3.2), which is linear in $\delta$ and $\boldsymbol{a}$, without squaring both sides of the inequality. Otherwise, squaring (3.1) will yield a quadratic inequality in $\delta$ and $\boldsymbol{a}$, which cannot be cast directly as a LP (or a SDP). Like the design of approximate linear-phase IIR filters, relaxation technique [10] has to be used and there is no guarantee of optimality. Equation (3.2) is not yet a LP problem because the constraints are bilinear function of $\delta$ and $\boldsymbol{a}\left(\boldsymbol{a}=\left[\boldsymbol{a}_{r}^{T}, \boldsymbol{a}_{i}^{T}\right]^{T}\right)$. To this end, we assume that the ripple $\delta$ is fixed and given at each iteration. Then, we solve the following optimization problem after discretizing the frequency variable $\omega$, in the band of interest $\Omega$, into $K$ points, $\omega_{k}$ for $k=1, \ldots, K$

$$
\begin{aligned}
\min _{m m b a} & \varepsilon \\
\text { subject to } & \varepsilon+\boldsymbol{v}_{r 1}^{T}\left(\omega_{k}\right) \cdot \boldsymbol{a}_{r}+\boldsymbol{v}_{i 1}^{T}\left(\omega_{k}\right) \cdot \boldsymbol{a}_{i} \geq 0 \\
& \varepsilon+\boldsymbol{v}_{r 2}^{T}\left(\omega_{k}\right) \cdot \boldsymbol{a}_{r}+\boldsymbol{v}_{i 2}^{T}\left(\omega_{k}\right) \cdot \boldsymbol{a}_{i} \geq 0
\end{aligned}
$$

where

$$
\begin{aligned}
\boldsymbol{v}_{r 1}\left(\omega_{k}\right) & =\left[\delta \cdot \boldsymbol{c}\left(\omega_{k}\right)+W\left(\omega_{k}\right) \cdot \boldsymbol{s}\left(\omega_{k}\right)\right] \\
\boldsymbol{v}_{i 1}\left(\omega_{k}\right) & =\left[\delta \cdot \boldsymbol{s}\left(\omega_{k}\right)-W\left(\omega_{k}\right) \cdot \boldsymbol{c}\left(\omega_{k}\right)\right] \\
\boldsymbol{v}_{r 2}\left(\omega_{k}\right) & =\left[\delta \cdot \boldsymbol{c}\left(\omega_{k}\right)-W\left(\omega_{k}\right) \cdot \boldsymbol{s}\left(\omega_{k}\right)\right] \\
\boldsymbol{v}_{i 2}\left(\omega_{k}\right) & =\left[\delta \cdot \boldsymbol{s}\left(\omega_{k}\right)+W\left(\omega_{k}\right) \cdot \boldsymbol{c}\left(\omega_{k}\right)\right], \quad k=1, \ldots, K
\end{aligned}
$$

and $\varepsilon$ is a fictitious optimization variable introduced to determine whether the given ripple $\delta$ can be achieved by the all-pass filter to be designed. If $\varepsilon \leq 0$, then, the given ripple can be achieved by the all-pass filter. Otherwise, the ripple is too small and it needs to be increased. To determine the minimum ripple of the all-pass filter when approximating the given specification, we combine the LP formulation in (3.3) with a bisection method, which will be explained in detail in Section III-B. The convergence of this algorithm is guaranteed. Further, linear and other SOCP constraints such as flatness at certain frequency can also be imposed, as we shall see in the design examples.

\section{B. Imposing Stability Constraints}

Our formulation is a modification of the method in [9] in that we first solve the problem in (3.3). If the given pole radius constraint is satisfied, then the solution obtained is the desired result for the given ripple. If not, the radii of those poles exceeding the prescribed pole radius will be reduced to obtain a feasible initial solution, and the procedure will be repeated. The following Rouche's theorem, which will be formulated as a set of SOCP constraints in the sequel, will then be invoked together with (3.3) to obtain a feasible solution. If $f(z)$ and $g(z)$ are analytic inside and on a closed contour $C$, and if $|g(z)|<|f(z)|$ on $C$, then $f(z)$ and $f(z)+g(z)$ have the same number of zeros inside $C$. In our problem, we set $f(z)$ and $g(z)$ as

$$
g(z)=z^{N} \Delta^{(i)}(z), \quad f(z)=z^{N} A^{(i)}(z)
$$

where $A^{(i)}(z)=a_{0}+a_{1} z^{-1}+\cdots+a_{N} z^{-N}$ is the denominator of the all-pass filter obtained at the $i$ th iteration and $\Delta^{(i)}(z)=$ $\Delta_{0}+\Delta_{1} z^{-1}+\cdots+\Delta_{N} z^{-N}$ is the incremental polynomial. The denominator at $(i+1)$ th iteration is thus $A^{(i+1)}=A^{(i)}+\Delta^{(i)}$. It can be seen that $f(z)$ and $g(z)$ are analytic inside and on $\left\{C_{\rho}:|z|=\rho<1\right\}$. Invoking Rouche's theorem, it follows that $f(z)+g(z)$ and $f(z)$ have the same number of zeros inside $C_{\rho}$ if $|g(z)|<|f(z)|,|z|=\rho$. Therefore, by enforcing this constraint, $A^{(i+1)}(z)$ and $A^{(i)}(z)$ will have the same numbers of zeros inside $C_{\rho}$. In other words, if $A^{(i)}(z)$ has all its roots inside $C_{\rho}$, so is $A^{(i+1)}(z)$. This allows us to impose the desired pole radius constraint. $|g(z)|<|f(z)|$ can also be written in terms of $\Delta^{(i)}(z)$ and $A^{(i)}(z)$ as

$$
\left|\Delta^{(i)}(z)\right|<\left|A^{(i)}(z)\right| \text {, on } C_{\rho}:|z|=\rho<1 \text {. }
$$

If the initial polynomial $A^{(0)}(z)$ is chosen with all its zeros inside $C_{\rho}$, then, with constraint (3.5), all subsequent polynomials $A^{(i)}(z), i=1,2, \ldots$, will have their zeros inside $C_{\rho}$. To formulate (3.5) as SOCP constraints, we substitute $z=e^{j \omega}$ for $-\pi \leq \omega \leq \pi$ and rewrite it as

$$
\begin{aligned}
&\left|\sum_{n=0}^{N} \Delta_{n} \rho^{-n} e^{-j n \omega}\right|<\left|\sum_{n=0}^{N} \boldsymbol{a}_{n} \rho^{-n} e^{-j n \omega}\right| \\
&-\pi \leq \omega \leq \pi \Leftrightarrow \chi(\omega)>\left[\beta_{r}^{2}(\omega)+\beta_{i}^{2}(\omega)\right]^{1 / 2}
\end{aligned}
$$

where

$$
\begin{aligned}
& \chi(\omega)=\left\{\left[\boldsymbol{a}_{r}^{(i) T} \boldsymbol{c}_{\rho}(\omega)+\boldsymbol{a}_{i}^{(i) T} \boldsymbol{s}_{\rho}(\omega)\right]^{2}\right. \\
& \left.+\left[\boldsymbol{a}_{r}^{(i) T} \boldsymbol{s}_{\rho}(\omega)-\boldsymbol{a}_{i}^{(i) T} \boldsymbol{c}_{\rho}(\omega)\right]^{2}\right\}^{1 / 2} \\
& \beta_{r}(\omega)=\Delta_{r}^{T} c_{\rho}(\omega)+\Delta_{i}^{T} s_{\rho}(\omega) \\
& \beta_{i}(\omega)=\Delta_{r}^{T} s_{\rho}(\omega)-\Delta_{i}^{T} c_{\rho}(\omega) \\
& \boldsymbol{c}_{\rho}(\omega)=\left[\begin{array}{llll}
1 & \rho^{-1} \cos \omega & \cdots & \rho^{-N} \cos N \omega
\end{array}\right]^{T}
\end{aligned}
$$




$$
\begin{aligned}
\boldsymbol{s}_{\rho}(\omega) & =\left[\begin{array}{llll}
0 & \rho^{-1} \sin \omega & \cdots & \rho^{-N} \sin N \omega
\end{array}\right]^{T} \\
a_{n}^{(i)} & =a_{r n}^{(i)}+j \cdot a_{i n}^{(i)} \\
\Delta_{n}^{(i)} & =\Delta_{r n}^{(i)}+j \cdot \Delta_{i n}^{(i)} \\
\boldsymbol{a}_{i}^{(\theta)} & =\left[\begin{array}{llll}
a_{\theta 0}^{(i)} & a_{\theta 1}^{(i)} & \ldots & a_{\theta N}^{(i)}
\end{array}\right]^{T} \\
\Delta_{\theta} & =\left[\begin{array}{llll}
\Delta_{\theta 0}^{(i)} & \Delta_{\theta 1}^{(i)} & \cdots & \Delta_{\theta N}^{(i)}
\end{array}\right]^{T}, \quad \theta=r, i .
\end{aligned}
$$

Since standard SOCP accepts strict inequalities rather than "equal and greater" inequalities of the form $\boldsymbol{d}_{i}^{T} \boldsymbol{x}+q_{i} \geq$ $\left\|A_{i} \boldsymbol{x}+\boldsymbol{c}_{i}\right\|_{2}$, the left-hand side of (3.6) is multiplied by a real constant $\alpha$ slightly less than 1 ( 0.98 in our simulation result) to give the following SOCP problem:

$$
\alpha \cdot \chi(\omega) \geq\|P(\omega) \cdot \Delta\|_{2}, \quad-\pi \leq \omega \leq \pi
$$

where

$$
P(\omega)=\left[\begin{array}{cc}
\boldsymbol{c}_{\rho}^{T}(\omega) & \boldsymbol{s}_{\rho}^{T}(\omega) \\
\boldsymbol{s}_{\rho}^{T}(\omega) & -\boldsymbol{c}_{\rho}^{T}(\omega)
\end{array}\right] \text { and } \boldsymbol{\Delta}=\left[\begin{array}{ll}
\boldsymbol{\Delta}_{r}^{T} & \boldsymbol{\Delta}_{i}^{T}
\end{array}\right]^{T} .
$$

$\alpha, 0<\alpha<1$ also helps to avoid the actual pole radius from exceeding the desired one, due to discretization of the Rouche constraints on a grid of frequencies. After discretizing the frequency variable $\omega$ in the band of interest into $L$ points, $\omega_{l}$ for $l=1, \ldots, L$, we obtain a set of SOCP constraints: $\alpha \cdot \chi\left(\omega_{l}\right) \geq$ $\left\|P\left(\omega_{l}\right) \cdot \Delta\right\|_{2}$, for $l=1, \ldots, L$. Finally, the overall design problem in terms of $\boldsymbol{\Delta}$ and $\boldsymbol{a}^{(i)}$ is

$$
\begin{aligned}
\min & \varepsilon \\
\text { subject to } & \varepsilon+\boldsymbol{v}_{r 1}^{T}\left(\omega_{k}\right) \cdot\left(\boldsymbol{a}_{r}^{(i)}+\boldsymbol{\Delta}_{r}\right) \\
& +\boldsymbol{v}_{i 1}^{T}\left(\omega_{k}\right) \cdot\left(\boldsymbol{a}_{i}^{(i)}+\boldsymbol{\Delta}_{i}\right) \geq 0 \\
\varepsilon & +\boldsymbol{v}_{r 2}^{T}\left(\omega_{k}\right) \cdot\left(\boldsymbol{a}_{r}^{(i)}+\boldsymbol{\Delta}_{r}\right) \\
& +\boldsymbol{v}_{i 2}^{T}\left(\omega_{k}\right) \cdot\left(\boldsymbol{a}_{i}^{(i)}+\boldsymbol{\Delta}_{i}\right) \geq 0 \\
\alpha & \cdot \chi\left(\omega_{l}\right) \geq\left\|P\left(\omega_{l}\right) \cdot \boldsymbol{\Delta}\right\|_{2}, \\
& k=1, \ldots, K \text { and } l=1, \ldots, L .
\end{aligned}
$$

Equation (3.8) is a combination of LP and SOCP problem, which can be readily solved, say using $S e D u M i$ [18]. Together with the bisection method, the all-pass filter with the minimum phase ripple and prescribed maximum pole radius can be obtained. The procedure is summarized in Table I. $\delta_{L}$ and $\delta_{U}$ are first initialized to some values specified by the user. In step 1 , the feasibility of the design problem is first tested by solving (3.3) with the maximum value of ripple $\delta_{U}$. If there is a solution and the maximum pole radius constraint is not violated, i.e the design problem is feasible for the given ripple $\delta_{U}$, we proceed to the bisection algorithm to determine the lowest possible ripple. If there is a solution but the pole radius constraints is violated, then the radii of those poles exceeding the prescribed pole radius will be reduced to obtain a feasible initial solution and (3.8) will be invoked with the pole radius constraints specified in (3.7). If there is a solution, we proceed to the bisection algorithm, otherwise, no solution is said to be found. Note, because the imposed constraint in (3.8) is only a sufficient condition, the solution obtained is no longer the global optimal solution as in (3.3). This is the reason why (3.3) is used whenever possible in Table I to avoid the inherent limitation of this constraint. In step 2 , a similar process is repeated until $\left|\delta_{L}-\delta_{U}\right|$ is smaller than a given tolerance. The ripple is set to $\delta=\left(\delta_{L}+\delta_{U}\right) / 2$ and (3.3)
TABLE I

SUMMARY OF PROPOSED DESIGN METHOD*

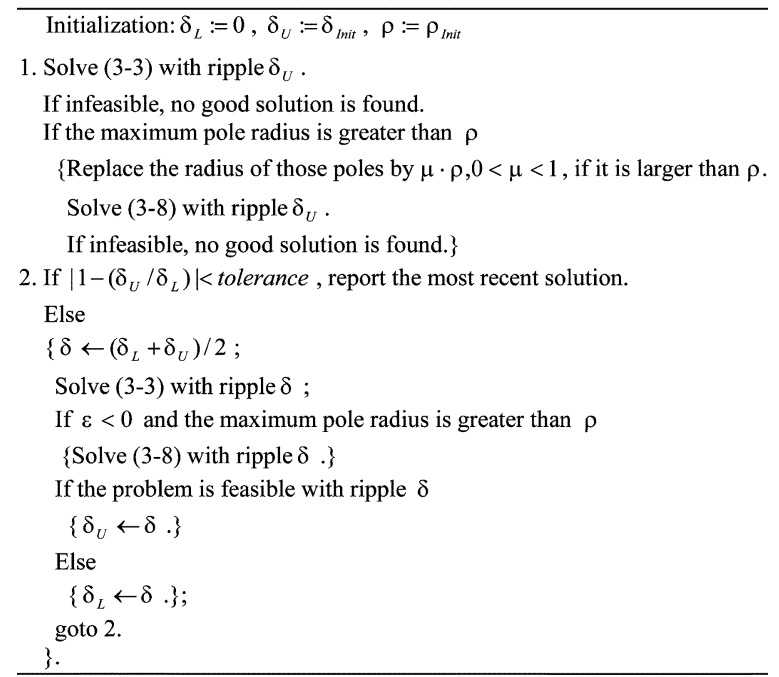

*The constant $\mu=0.97$ is used to change the poles back to inside but not on the stability contour. the term "feasible" means that the design problem can be solved with the given ripple $\delta$ and pole radius constraint.

is solved for $\varepsilon$. If the solution of $\varepsilon$ is greater than zero, then the given ripple $\delta$ is too small (infeasible) and it will be set to the new lowerbound, i.e., $\delta_{L}=\delta$. On the other hand, if $\varepsilon \leq 0$, the maximum pole radius constraint will be tested and (3.8) will be invoked with the given ripple, if the constraint is violated. If the design problem is feasible for the given ripple, the upper bound will be reduced to $\delta_{U}=\delta$. Otherwise, the lowerbound will be increased to $\delta_{L}=\delta$. Since the difference between the two bounds of the ripples is reduced by half at each iteration, the minimum achievable ripple and the filter coefficients will be found eventually when this difference goes to zero. Thus, the convergence of the algorithm is also guaranteed.

\section{DESIGN EXAMPLES}

We now present an example of half-band filters (HBFs) with the pole radius constraint. All the SOCP problems were solved by the SeDuMi Toolbox. For comparison purposes, some other examples are summarized in Table II are chosen from conventional design methods previously reported in [2], [4], [13], [14]. The $\alpha$ in (3.7) is chosen as 0.98 .

The transfer function of the HBF is given by $H(z)=$ $(1 / 2)\left(z^{-2 N}+z^{-1} \beta\left(z^{2}\right)\right)$, where $\beta(z)$ is an all-pass digital filter. The desired response of $\beta(z)$ is given by $\beta_{d}\left(e^{j \omega}\right)=e^{-j(N-0.5) \omega}, \omega \in\left[0,2 \omega_{p}\right]$, where $\omega_{p}$ is the passband cutoff frequency of the HBF. The order $N$ and the cutoff frequency of the all-pass filter are, respectively, set to 8 and $\omega_{p}=0.4 \pi$. This is identical to [2, Ex. 2]. The number of points $K$ in the interested bands and the number of points $L$ on the stability contour are set to 200 . The optimization procedure needs 23 iterations and 24 seconds in a Pentium4 $1.8-\mathrm{GHz}$ computer. The tolerance of the ripple $\delta$ is set to 0.001 . The maximum pole radius $\rho$ is set to three different values: $0.98,0.8$ and 0.78 to study their effects on the frequency characteristics. Fig. 1(a) shows the frequency responses of the three HFBs so obtained. The stopband attenuations are, respectively, 72.47, 66.94, and $60.16 \mathrm{~dB}$ for $\rho=0.98,0.8$, 
TABLE II

COMPARISON OF VARIOUS AlgORITHMS *

\begin{tabular}{|c|c|c|c|c|c|c|c|c|c|c|}
\hline & Approach & $\Omega_{p} / \pi$ & $\Omega_{s} / \pi$ & $\rho_{c}$ & $\rho_{\max }$ & $\delta_{p}(\mathrm{~dB})$ & $A_{s}(\mathrm{~dB})$ & Phase error & $\mathrm{SOC}^{\circledR}$ & INIT $^{*}$ \\
\hline \multirow{5}{*}{$\begin{array}{l}\text { Half-band filter } \\
\text { (Design } \\
\text { Example) }\end{array}$} & \multirow{3}{*}{$\mathrm{SOCP}^{\#}$} & \multirow{5}{*}[0,0.4]{} & \multirow{5}{*}[0.6,1]{} & 0.98 & 0.81 & $2.54 \times 10^{-7}$ & 72.47 & $1.5 \times 10^{-4} \pi$ & \multirow{3}{*}{ Yes } & \multirow{3}{*}{ No } \\
\hline & & & & 0.80 & 0.80 & $8.78 \times 10^{-7}$ & 66.94 & $3.0 \times 10^{-4} \pi$ & & \\
\hline & & & & 0.78 & 0.78 & $4.18 \times 10^{-6}$ & 60.16 & $6.1 \times 10^{-4} \pi$ & & \\
\hline & Remez [2] & & & N/A & $\mathrm{N} / \mathrm{A}$ & N/A & 72.5 & $5 \times 10^{-4} \mathrm{rad}^{*}$ & No & Yes \\
\hline & eigen-filter [3] & & & $\mathrm{N} / \mathrm{A}$ & $\mathrm{N} / \mathrm{A}$ & N/A & 71.2 & $6 \times 10^{-4} \mathrm{rad}^{*}$ & No & Yes \\
\hline \multirow{4}{*}{$\begin{array}{c}\text { AP-based } \\
\text { multiband filter }\end{array}$} & SOCP & \multirow{2}{*}{$\begin{array}{l}{[0,0.4],} \\
{[0.8,1]}\end{array}$} & \multirow{2}{*}{$\begin{array}{c}{[0.45,0.75]} \\
\text { (40dB at } \\
\text { bands } \\
{[0.45,0.5]} \\
{[0.7,0.75)}\end{array}$} & N/A & $\mathrm{N} / \mathrm{A}$ & $2 \times 10^{-6}$ & 64.35 & $8 \times 10^{-4} \pi$ & Yes & No \\
\hline & Remez-type [13] & & & $\mathrm{N} / \mathrm{A}$ & $\mathrm{N} / \mathrm{A}$ & $4.34 \times 10^{-6}$ & 60 & N/A & No & Yes \\
\hline & SOCP & \multirow{2}{*}{$\begin{array}{c}{[0,0.3],} \\
{[0.65,0.75]}\end{array}$} & \multirow{2}{*}{$\begin{array}{c}{[0.35,0.6]} \\
{[0.8,1]}\end{array}$} & N/A & N/A & 0.2 & 60.41 & $9 \times 10^{-4} \pi$ & Yes & No \\
\hline & Remez-type [13] & & & N/A & N/A & 0.2 & 60 & $\mathrm{~N} / \mathrm{A}$ & No & Yes \\
\hline \multirow{2}{*}{$\begin{array}{c}\text { Approximately } \\
\text { linear phase IIR } \\
\text { complimentary } \\
\text { filter }\end{array}$} & SOCP $^{\#}$ & \multirow{2}{*}[0,0.4]{} & \multirow{2}{*}[0.6,1]{} & 0.98 & 0.78 & $9.15 \times 10^{-4}$ & 36.76 & $7.4 \times 10^{-3} \pi$ & Yes & No \\
\hline & Eigenvalue[4] & & & N/A & N/A & N/A & $36.7^{*}$ & $7.4 \times 10^{-3} \pi^{*}$ & No & Yes \\
\hline \multirow{2}{*}{$\begin{array}{c}\text { All-pass filters } \\
\text { with Complex- } \\
\text { valued } \\
\text { coefficients }\end{array}$} & SOCP & \multirow{2}{*}[0,2]{} & \multirow{2}{*}{ N/A } & 0.98 & 0.81 & N/A & N/A & $0.1013 \mathrm{rad}$ & Yes & No \\
\hline & $\begin{array}{c}\text { Remez-exchange } \\
{[14]}\end{array}$ & & & $\mathrm{N} / \mathrm{A}$ & N/A & $\mathrm{N} / \mathrm{A}$ & N/A & $0.1013 \mathrm{rad}$ & No & Yes \\
\hline \multirow[b]{2}{*}{ IIR filter pairs } & SOCP & \multirow[b]{2}{*}[0,2]{} & \multirow{2}{*}[0.4,1]{} & 0.98 & 0.85 & $9.4 \times 10^{-5}$ & 37.81 & $5 \times 10^{-3} \mathrm{rad}$ & Yes & No \\
\hline & $\begin{array}{c}\text { Remez-exchange } \\
{[14]}\end{array}$ & & & N/A & $\mathrm{N} / \mathrm{A}$ & N/A & $37.8^{*}$ & N/A & No & Yes \\
\hline \multirow[b]{2}{*}{ QMF } & SOCP & \multirow[b]{2}{*}[0,0.4]{} & \multirow[b]{2}{*}[0.6,1]{} & 0.98 & 0.78 & $5 \times 10^{-6}$ & 59 & $4 \times 10^{-4} \mathrm{rad}$ & Yes & No \\
\hline & $\begin{array}{c}\text { Remez-exchange } \\
{[14]}\end{array}$ & & & N/A & N/A & N/A & $59^{*}$ & $4 \times 10^{-4} \mathrm{rad}^{*}$ & No & Yes \\
\hline
\end{tabular}

$* \Omega_{p}$ and $\Omega_{s}$ denote passband(s) and stopband(s) of filters, $\rho_{c}$ is radius of contour $C$, $\rho_{\max }$ is maximum pole radius of filter, $\delta_{p}$ and $A_{s}$ are, respectively, passband ripple and stopband attenuation of filters. @ : SOC means imposing additional SOCP constraints and INIT means whether initial guess for initial extremal frequencies is required. $N / A$ means that result is either not available or not applicable, * means that results are gauged from figures in corresponding references because they are not given explicitly, \# indicates that results for flatness constraints are not shown here for sake of presentation, please refer to [23] for details.

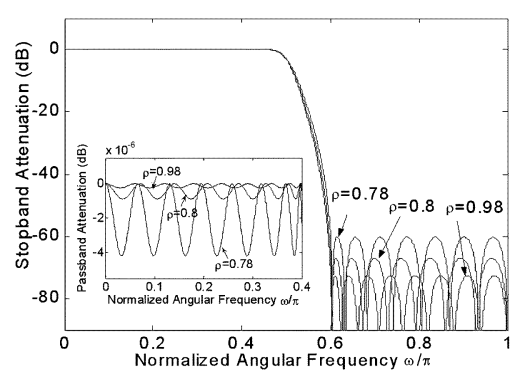

(a)

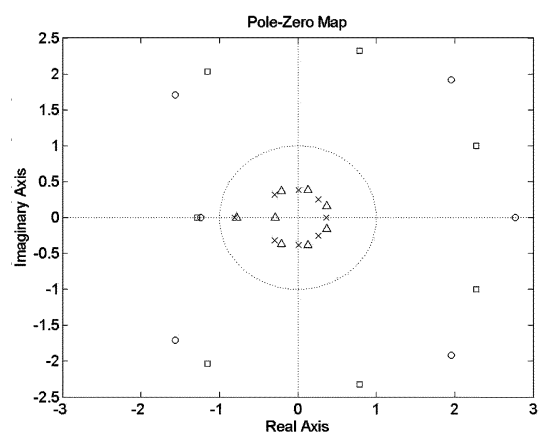

(c)

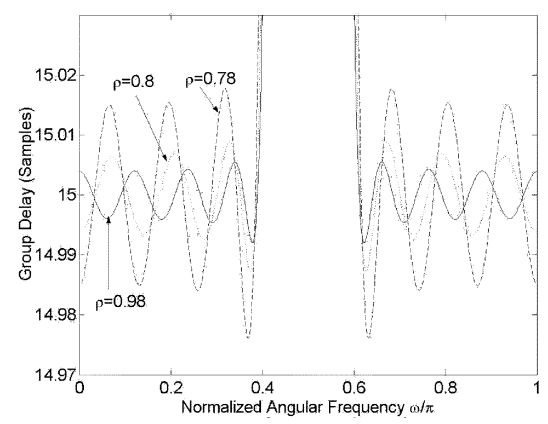

(b)

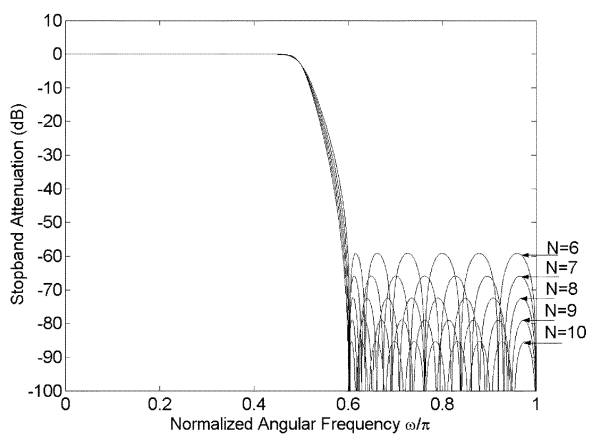

(d)

Fig. 1. Design results. (a) Magnitude response. The smaller figure is the passband responses. (b) Group delay. (c) Pole-zero plots of $\rho=0.98$ and $\rho=0.78$. The crosses and circles are, respectively, the poles and zeros of the filter with $\rho=0.78$. (d) Frequency response for different values of $N$.

and 0.78 . It can be seen that there is a tradeoff between the stability margin and the amount of stopband attenuation: the smaller the maximum pole radius (larger stability margin), the smaller the stopband attenuation. A similar situation occurs in the passband and group-delay responses as shown in Fig. 1(a) and (b). It can be seen that the all-pass-based HBF has an approximate linear phase response in the passband. Fig. 1(c) shows the pole-zero plots of the two filters with maximal pole 
radii set to 0.98 and 0.78 (The pole-zero plot for the filter with maximum pole radius set to 0.8 is omitted here due to page limitation). The resulting maximum pole radii are $0.81,0.8$, and 0.78 , respectively. All of them satisfy the prescribed pole radius constraints, showing the effectiveness of the constraints imposed by (3.7). The best performance (72.47-dB attenuation) is obtained with a pole constraint of 0.98 , and the resulting maximum pole radius is 0.81 . This is very close to $72.5 \mathrm{~dB}$ that was reported to be the optimal solution in [2]. The stopband attenuation obtained by the eigen-filter approach is $71.2 \mathrm{~dB}$ [3]. The design results suggest that the optimal all-pass filter has a maximum pole radius of around 0.81 . Therefore, when $\rho$ is chosen as 0.98 , the pole constraint does not have any effect visually on the stopband attenuation. On the other hand, if the pole constraint is smaller than 0.81 , the ripple will be increased to meet the smaller prescribed pole radius constraint. All-pass filters with different order $N$ were also designed for the same specification of cutoff frequencies with stability constraint only, and the resulting stopband attenuation is plotted in Fig. 1(d). It can be seen that the difference in stopband attenuation between successive values of $N$ is rather large. Therefore, the stopband attenuation can be traded for better stability margin.

To impose a prescribed flatness, say $2 r_{0}+1$ zeros at $\omega=\pi$, its coefficients should satisfy: $\sum_{n=0}^{N}\left\{a_{n} \cdot(1-4 n)^{2 i-1}\right\}=0$ for $i=1,2, \ldots, r_{0}$. Design results, not shown here due to page limitation, show that the desired number of zeros can be imposed on the HBF. This problem was not addressed in traditional design techniques [2]-[8], [13], [14].

We summarize all the results in this brief and some other related examples in Table II. It is noted that the proposed method can also be applied to all-pass-based multiband filters [13], allpass filters with complex-valued coefficients, and noninteger delay IIR filter pair by connecting two all-pass filters in parallel . For comparison purpose, we designed a noninteger delay IIR filter pair with the same specification in [4]. The orders of the all-pass filters are 11 and 10, respectively. The design results are almost identical to those reported in [4], but no initial values of the extremal frequency points are required and a prescribed magnitude flatness can be incorporated. Significant improvements are also observed in the all-pass-based multiband filters. Design examples on several all-pass filters with complex-valued coefficients were also listed in Table II. These examples also suggest that both our algorithm and the methods in [14] and [4] can find the optimal unconstrained minimax solution. Again, our method has the freedom of imposing linear and nonlinear SOCP constraints and it is unnecessary to specify the initial extremal frequencies. Interested readers are referred to [16] for more information.

\section{CONCLUSION}

A new method for designing digital all-pass filters with a minimax design criterion using LP is presented. Unlike other all-pass filter design methods, additional linear constraints can be readily incorporated. The convergence of the algorithm is guaranteed. Nonlinear constraints such as the pole radius constraint and hence the stability margin of the filters can be formulated as additional SOCP constraints using Rouche's theorem.
The effectiveness of the proposed method is demonstrated by several design examples.

\section{REFERENCES}

[1] P. A. Regalia, S. K. Mitra, and P. P. Vaidyanathan, "The digital all-pass filter: A versatile signal processing building block," Proc. IEEE, vol. 76, no. 1, pp. 19-37, Jan. 1988.

[2] M. Lang, "All-pass filter design and applications," IEEE Trans. Signal Process., vol. 46, pp. 2505-2514, Sep. 1998.

[3] T. Q. Nguyen, T. I. Laakso, and R. D. Koilpillai, "Eigenfilter approach for the design of all-pass filters approximating a given phase response," IEEE Trans. Signal Process., vol. 42, pp. 2257-2263, Sep. 1994.

[4] X. Zhang and H. Iwakura, "Design of IIR digital all-pass filters based on eigenvalue problem," IEEE Trans. Signal Process., vol. 47, no. 2, pp. 554-559, Feb. 1999.

[5] C. K. Chen and J. H. Lee, "Design of digital all-pass filters using a weighted least squares approach," IEEE Trans. Circuits Syst. II, Analog Digit. Signal Process., vol. 41, no. 5, pp. 346-350, May 1994.

[6] S. C. Pei and J. J. Shyu, "Eigenfilter design of 1-D and 2-D IIR digital all-pass filters," IEEE Trans. Signal Process., vol. 42, no. 4, pp. 996-968, Apr. 1994

[7] M. Ikehara, H. Tanaka, and H. Kuroda, "Design of IIR digital filters using all-pass networks," IEEE Trans. Circuits Syst. II, Analog Digit. Signal Process., vol. 41, no. 3, pp. 231-235, Mar. 1994.

[8] Z. Jing, "A new method for digital all-pass digital filter design," in IEEE Trans. Acoust., Speech, Signal Process., vol. ASSP-35, Nov. 1987, pp. $1557-1564$.

[9] M. C. Lang, "Least-Squares design of IIR filters with prescribed magnitude and phase responses and a pole radius constraint," IEEE Trans. Signal Process., vol. 48, no. 11, pp. 3109-3121, Nov. 2000.

[10] W. S. Lu, "Design of stable minimax IIR digital filters using semidefinite programming," in Proc. Int. Symp. Circuits Syst. (ISCAS'00), Geneva, Switzerland, May 28-31, 2000, pp. I-355-358.

[11] M. S. Lobo, L. Vandenberghe, S. Boyd, and H. Lebret, "Applications of second-order cone programming," Lin. Algeb. Applicat., vol. 248, pp. 193-228, 1998.

[12] R. V. Churchill and J. W. Brown, Complex Variables and Applications. New York: McGraw-Hill, 1984.

[13] T. Saramaki and M. Renfors, "A Remez-type algorithm for designing digital filters composed of all-pass sections based on phase approximations," in Proc. 38th Midwest Symp. Circuits Syst., vol. 1, Aug. 1995, pp. $571-575$.

[14] M. Ikehara, M. Funaishi, and H. Kuroda, "Design of complex all-pass networks using Remez algorithm," IEEE Trans. Circuits Syst. II, Analog Digit. Signal Process., vol. 39, no. 8, Aug. 1992.

[15] M. Renfors and T. Saramaki, "A class of approximately linear phase digital filters composed of all-pass subfilters," in Proc. Int. Symp. Circuits Syst. (ISCAS'86), 1986, pp. 678-681.

[16] D. P. Scholnik, "Mixed-norm FIR filter optimization using second-order cone programming," in Proc. Int. Symp. Circuits Syst. (ISCAS'02), vol. 2, 2002, pp. 1525-1528.

[17] W. S. Lu, "Optimal design of FIR frequency-response-masking filters using second-order cone programming," in Proc. Int. Symp. Circuits Syst. (ISCAS'03), vol. 3, May 2003, pp. 878-881.

[18] J. F. Sturm, "Using SeDuMi 1.02, a MATLAB toolbox for optimization over symmetric cones," Optim. Meth. Softw., vol. 11-12, pp. 625-653, 1999.

[19] B. Alkire and L. Vandenberghe, "Convex optimization problems involving finite autocorrelation sequences," Math. Progr. Ser. A, vol. 93, no. 3, pp. 331-359, 2002.

[20] T. N. Davidson, Z. Q. Luo, and J. F. Sturm, "Linear matrix inequality formulation of spectral mask constraints with applications to FIR filter design," IEEE Trans. Signal Process., vol. 50, no. 11, pp. 2702-2715, Nov. 2002.

[21] S.-P. Wu, S. Boyd, and L. Vandenberghe, "FIR filter design via semidefinite programming and spectral factorization," in Proc. 5th Conf. Decision Contr., Kobe, Japan, Dec. 1996, pp. 271-276.

[22] C. K. S. Pun and S. C. Chan, "The minimax design of digital all-pass filters with prescribed pole radius constraint using semidefinite programming (SDP)," in Proc. Int. Conf. Acoust., Speech, Signal Process. (ICASSP'03), vol. VI, 2003, pp. 413-416.

[23] S. C. Chan and H. H. Chen, "The design of digital all-pass filters using second-order cone programming (SOCP)," Dep. Elect. Electron. Eng., Univ. of Hong Kong, Hong Kong, Intern. Rep., 2003. 